\title{
Extended coronal emission lines in active galactic nuclei
}

\author{
Alberto Rodríguez-Ardila ${ }^{1}$, Almudena Prieto ${ }^{2}$ and Sueli M. Viegas ${ }^{3}$ \\ ${ }^{1}$ LNA/MCT, Rua dos Estados Unidos 154, Itajubá, MG, Brazil email: aardila@lna.br \\ ${ }^{2}$ Max Plank Institute, Heidelberg, Germany \\ ${ }^{3}$ IAG-Universidade de São Paulo, SP, Brazil
}

\begin{abstract}
VLT and NTT spectra are used to examine the nuclear and extended coronal line emission in a sample of well-known Seyfert 1 and 2 galaxies. The excellent spatial resolution obtained with VLT allowed us to map [Si vi] $1.963 \mu \mathrm{m}$ and [Si VII] $2.48 \mu \mathrm{m}$ on scales of up to 20 pc. Coronal line emission, extended to distances of $\sim 10^{2} \mathrm{pc}$, is detected in some of the lines analyzed, particularly in [Fex] 6374 $\AA$, [Fe XI] $7891 \AA$, and [Si VII] $2.48 \mu \mathrm{m}$. Most coronal lines are strongly asymmetric towards the blue and broader than low-ionization lines. This result is particularly important for Circinus, where previous observations have failed to detect larger widths for high-ionization lines. Photoionization models are used to investigate the physical conditions and continuum luminosities necessary to produced the observed coronal emission. We found that an ionization parameter $U>0.10$ is necessary to reproduce the observations, although the clouds should be located at a distance $<30 \mathrm{pc}$ from the central engine.
\end{abstract}

\section{Introduction}

Coronal lines (CL) are collisionally excited forbidden transitions within low-lying levels of highly ionized species (IP>100 eV). They can be formed either by a hard UV continuum (Marconi et al. 1996; Ferguson et al. 1997), a very hot collisionally ionized plasma (Viegas-Aldrovandi \& Contini 1989), or a combination of both processes (Contini et al. 1998). Due to the high energies involved in their production, the detection of CL is taken as an unambiguous signature of nuclear activity and can provide clues on the UV to soft X-ray spectral energy distribution of the active nucleus (Prieto et al. 2002).

Observationally, CL are blueshifted relative to the systemic velocity of the host galaxy $(\Delta \mathrm{V} \sim 500-800 \mathrm{~km} / \mathrm{s})$ and broader than low ionization lines (Penston et al. 1984). This has led to the idea that CL are associated to outflows (Erkens et al. 1997) and formed in a separate region (termed as coronal line region, CLR) located between the classical narrow line region (NLR) and broad line region. However, both the physical conditions of the coronal gas and the size of the emitting region are matters of strong controversy (Erkens et al. 1997; Nazarova et al. 1999). Published results usually rely on measurements made on just a few CLs and information about the size of their emitting regions, on the same target, is very scarce.

With this in mind, the goals of this work are: (i) Observe a sample of well-known AGN in order to measure the size of the emitting region for different coronal lines; (ii) model the observe emission to obtain clues on the physical conditions of the CLR; (iii) study the kinematics of the coronal gas and compare it to that of other NLR lines.

\section{Main results}

A sub-sample of the observed data, collected using VLT/ISAAC at ESO for NGC 1068 and Circinus, is presented in Figure 1. The spatial scale of the detector $(0.15 \% / \mathrm{pix})$, 

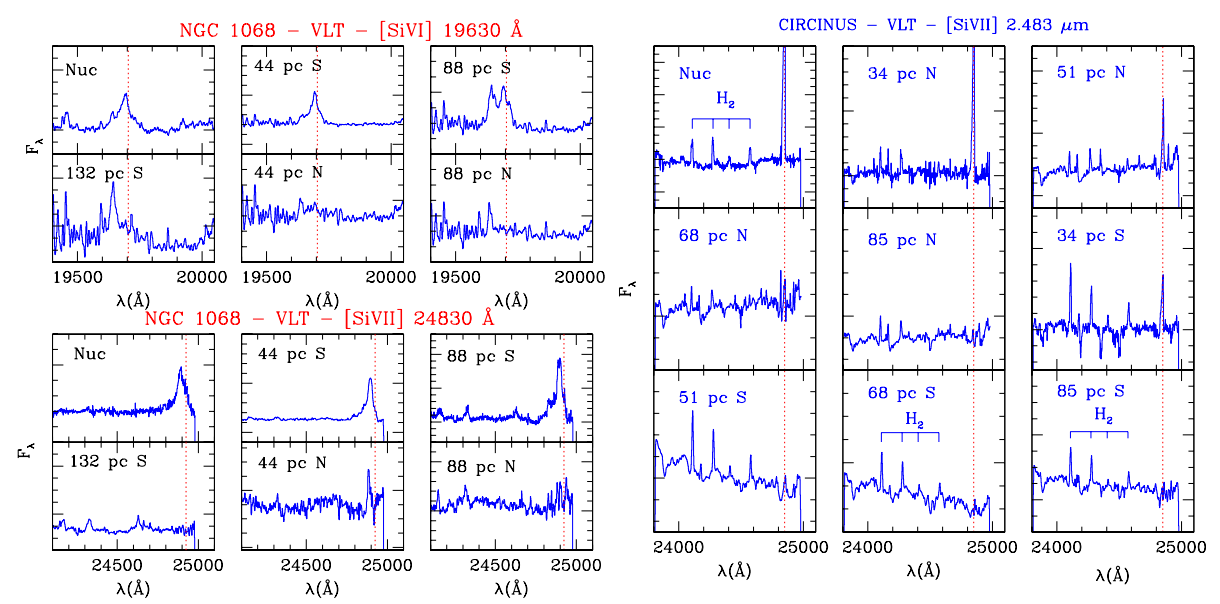

Figure 1. Observed VLT/ISAAC NIR spectra of NGC 1068 (left) and Circinus (right). We indicate, in each panel, the distance from the centre of the AGN to which the spectrum corresponds. The dotted line marks the centroid position of the CL. $\mathrm{F}_{\lambda}$ is in arbitrary units.

combined with the good seeing during the observations $\left(\sim 0.6^{\prime \prime}\right)$ allowed us to map gas emission at a spatial resolution of 20 pc. The slit was oriented north-south in both configurations.

The right panel of Figure 1 shows that in Circinus the [Si VII] line extends from the unresolved nucleus $(\mathrm{R}<17 \mathrm{pc})$, up to $\sim 70 \mathrm{pc}$ to the north and $34 \mathrm{pc}$ to the south, implying a total size of $\sim 100 \mathrm{pc}$ for the [Si VII] emitting region. In contrasts, [Si VI] (not shown in the Figure 1 ) is limited to the central $\sim 70 \mathrm{pc}$. These values, however, are larger than those derived by Oliva et al. (1994), who estimated that the size of the CLR in Circinus was only $\sim 10 \mathrm{pc}$. Due to the better sensitivity of our data, we show that the coronal gas extends farther out from the centre than previously found. For comparison, note that the $\mathrm{H}_{2}$ lines in Figure 1 are detected in all regions where spectra were extracted.

In NGC 1068, both [Si VI] and [Si vII] extends from the unresolved nucleus to $90 \mathrm{pc}$ south and up to $40 \mathrm{pc}$ north (bottom and upper left panels of Figure 1). These values, however, are significantly lower than that of $\sim 300 \mathrm{pc}$ to the south and $200 \mathrm{pc}$ to the north derived from NICMOS [Si vI] imaging by Thompson et al. (2001). Note, however, that the Thomson et al (2001) data may be misleading because of contamination of [Si VI] $1.963 \mu \mathrm{m}$ by $\mathrm{H}_{2} 1.957 \mu \mathrm{m}$, easily seen in Figure 1, where both lines are heavily blended. At $88 \mathrm{pc}$ from the nucleus, [Si VI] $1.963 \mu \mathrm{m}$ and $\mathrm{H}_{2} 1.957 \mu \mathrm{m}$ have similar strengths and at distances larger than $120 \mathrm{pc}$, only $\mathrm{H}_{2}$ is detected.

Overall, our data show that all CL are emitted in the inner $100 \mathrm{pc}$ of the active nucleus. When compared to low-ionization and molecular lines, we found that the emitting region of the latter two set of lines extends to much larger distances $(\mathrm{R}>500 \mathrm{pc})$. This result rules out the hypothesis of Ferguson et al. (1997) of a a low-density CLR extending up to $1 \mathrm{kpc}$ from the active nucleus but also rules out very compact CLR with upper limits of a few parsecs.

\section{Are pure photoionization driving the CL emission?}

We tested the possibility of the CL being emitted from distances of up to several tens of parsecs from the central engine assuming pure photoionization. For this purpose, we run models with the AANGABA code (Gruenwald \& Viegas 1992). The adopted 

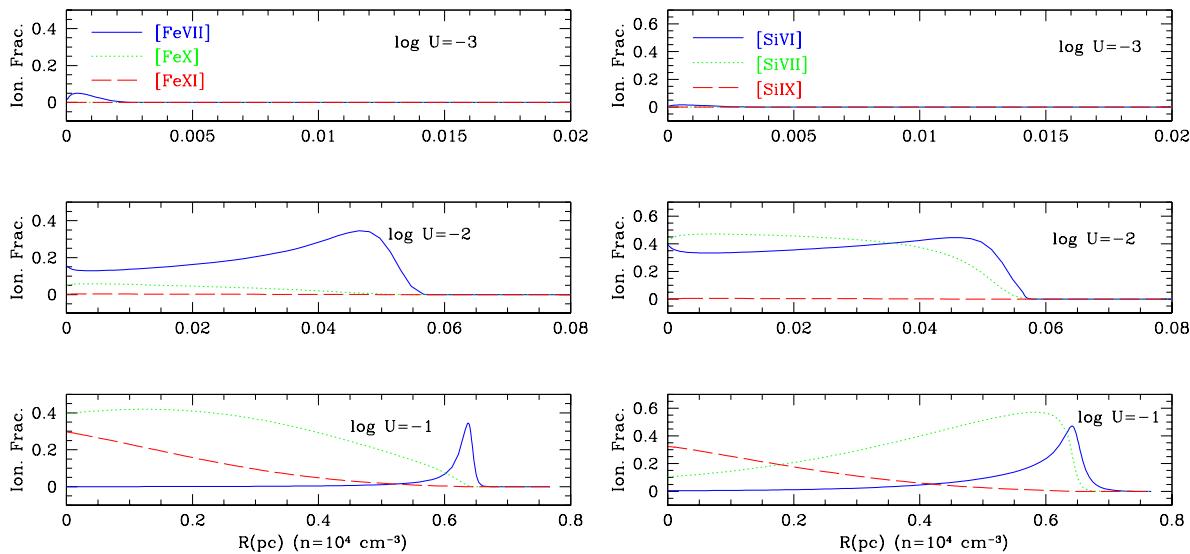

Figure 2. Ionic abundance for the Fe ions (left) and Si (right) versus the distance within the cloud for $L_{\text {ion }}=10^{43.5} \mathrm{erg} \mathrm{s}^{-1}$. Typical cloud sizes are $\sim 1 \mathrm{pc}$.

spectral energy distribution (SED) was that suggested in Fig. 7 of Oliva et al. (1999) for Circinus. Two values of luminosity of the ionizing radiation were adopted: $L_{\text {ion }}=10^{43.5}$ erg s${ }^{-1}$ and $L_{\text {ion }}=10^{44.5} \mathrm{erg} \mathrm{s}^{-1}$, suggested by Ferguson et al. (1999) and Oliva et al. (1999), respectively. The number of ionizing photons, $Q_{\mathrm{H}}$, provided by these two SEDs are $2.5 \times 10^{53} \mathrm{~s}^{-1}$, and $2.5 \times 10^{54} \mathrm{~s}^{-1}$. The density was varied from $10^{2} \mathrm{~cm}^{-3}$ to $10^{6} \mathrm{~cm}^{-3}$. Three values for the ionization parameter $U$ were employed: $10^{-3}, 10^{-2}$ and $10^{-1}$.

Figure 2 shows the distribution of the ionic fraction of iron and silicon versus the size of the emitting cloud for the three values of $U$ employed. Note that $\mathrm{R}$, equivelent to the cloud size, is measured from the side of the cloud facing the ionizing radiation to the outer edge. The value of density that best reproduces the observations is $n_{\mathrm{H}}=10^{4} \mathrm{~cm}^{-3}$, in accord to that of $0.5 \times 10^{4} \mathrm{~cm}^{-3}$ determined for the CLR from the [Nev] $14.3 \mu \mathrm{m} /$ $24.3 \mu \mathrm{m}$ line ratio with $I S O$ (Moorwood et al. 1996).

The results show that an $U \geqslant 0.10$ is necessary in order to have clouds with an ionization structure similar to that observed -i.e., simultaneous presence of [Si VI], [Si VII], and [Fe VII] through [Fe XI]. In addition, for the parameters adopted, the emitting clouds must be located within 8 to $30 \mathrm{pc}$ from the central engine. Coronal emission located farther out from the source would requiere $L_{\mathrm{ion}}>10^{44} \mathrm{erg} \mathrm{s}^{-1}$. More detailed modeling of our data can be found in Rodríguez-Ardila et al. (2004, in preparation).

\section{Kinematics of the coronal gas}

The kinematics of the coronal gas was studied by comparing the emission line profiles of low and high excitation lines. Figure 3 shows the results obtained for Circinus and NGC 3783.

As can be easily seen, the coronal lines in both objects are highly asymmetric towards the blue and broader than low-ionization lines. Previous works on Circinus (Oliva et al. 1994, 1999) had failed to detect any significant variation between the width and the IP of the forbidden lines. In fact, Moorwood et al. (1996) had reported narrower widths in high excitation lines than in lower excitation lines. The VLT/ISAAC and NTT/EMMI spectra of Figure 3 remove this ambiguity and show the presence of coronal gas with velocities close to $500 \mathrm{~km} \mathrm{~s}^{-1}$ in Circinus. Blue asymmetric profiles are also observed in NGC 3783. Note, however, that the line profile of [Fe XI] implies radial velocities of up to 

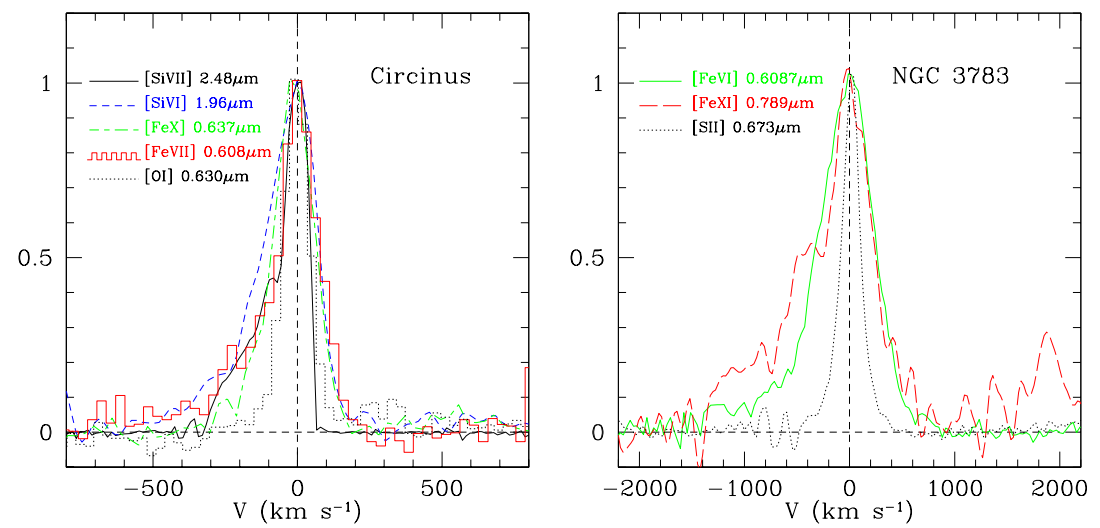

Figure 3. Comparison of low ionization and coronal emission line profiles, in velocity space, for Circinus (left) and NGC 3783 (right)

$1500 \mathrm{~km} \mathrm{~s}^{-1}$, suggesting as in Circinus, that part of the gas must arise in outflows very close to the central engine. Of the six objects studied, only NGC 1068 shows no difference between the FWHM of low and high ionization lines.

\section{Summary}

EMMI/NTT and ISAAC/VLT spectra are used to determine the size of the CLR in a sample of well-known Seyfert 1 galaxies by means of the simultaneous observation of [Fe VII], [Fex], [FexI], [Si vI] and [Si viI] lines. Our data show that all coronal lines are emitted from the unresolved nucleus to distances of up to $80 \mathrm{pc}$. We showed that the coronal emission region can arise from gas photoionized by radiation from the central engine if $U \geqslant 0.1, n_{\mathrm{e}} \sim 10^{4} \mathrm{~cm}^{-3}$, solar abundances, cloud sizes of $\sim 1 \mathrm{pc}$ and distances from the central source of up to $30 \mathrm{pc}$. The analysis of the coronal emission line profiles shows that they are highly asymmetric towards the blue, even in Circinus, where previous observations had measured widths of the order of $100 \mathrm{~km} \mathrm{~s}^{-1}$. For this object, we found coronal gas with velocities of up to $400 \mathrm{~km} \mathrm{~s}^{-1}$, suggesting that shocks must probably contribute to the observed emission.

\section{References}

Contini, M., Prieto, M. A., \& Viegas, S. M. 1998, ApJ, 505, 621

Erkens, U., Appenzeller, I., \& Wagner, S. 1997, A\&A, 323, 707

Ferguson, J. W., Korista, K. T., \& Ferland, G. J. 1997, ApJS, 110, 287

Gruenwald, R., \& Viegas, S. M. 1992, ApJS, 78, 153

Marconi, A., van der Werf, P. P., Moorwood, A. F. M., \& Oliva, E. 1996, A\&A, 315, 335

Moorwood, A. F. M., et al. 1996, A\&A, 315, L109

Nazarova, L. S., O'Brien, P. T., \& Ward, M. J. 1999, A\&A, 343, 682

Oliva, E., Marconi, A., \& Moorwood, A. F. M. 1999, A\&A, 342, 87

Oliva, E., Salvati, M., Moorwood, A. F. M., \& Marconi, A. 1994, A\&A, 288, 457

Penston, M., Fosbury, R. A. E., Boksenberg, A., Ward, M. J., \& Wilson, A. S. 1984, MNRAS, 208,347

Prieto, M. A., Pérez Garcia, A. M., \& Rodríguez Espinoza, J. M. 2002, MNRAS, 329, 309

Thompson, R., Chary, R.-R., Corbin, M. R, \& Epps, H. 2001, ApJ, 558, L97

Viegas-Aldrovandi, S., \& Contini, M. 1989, ApJ, 339, 689 